US WURK LXVIII (2019), p.

\title{
[1476] Die Sprache der erstmaligen Verschriftlichung der 17 Küren und der 24 Landrechte - Altfriesisch oder Latein?
}

\author{
Ann-Kathrin Reinders
}

\section{Einleitung ${ }^{1}$}

Auf dem Gebiet der Altfrisistik hat die Untersuchung des Verhältnisses der überlieferten altfriesischen und lateinischen Texte zueinander stets eine besondere Stellung eingenommen. Dabei ist trotz einer Untersuchung seit dem Ende des 19. Jahrhunderts immer noch umstritten, ob die einschlägigen älteren Rechtstexte auf dem damaligen friesischen Gebiet ursprünglich auf Latein oder auf (Alt-)Friesisch ,entworfen“2 wurden (Popkema 2014: 369³ Bremmer 2009: 9).

Die Untersuchung dieser Frage erfolgt typischerweise an den 17 Küren und den 24 Landrechten in den altfriesischen und lateinischen Versionen, die in den zwei Hunsingoer Handschriften enthalten sind. Die Texte der 17 Küren und der 24 Landrechte werden als die zentralen Rechtsquellen des mittelalterlichen friesischen Rechts gesehen (Bremmer 2014: 5).

Bisher wurden in der Literatur zumeist lediglich einzelne Argumentationsstränge aufgezeigt. Nur zu Beginn der Diskussion, als noch wenige Meinungen zu berücksichtigen waren, wurden diese teils gegenübergestellt. $\mathrm{Zu}-$ letzt ist Algra (1966, 1991) im Detail auf die von Heck (1931) gelieferte Begründung für eine Priorität des lateinischen Textes eingegangen. Teilweise finden sich Übersichten verschiedener Meinungen, dies jedoch ebenfalls lediglich im Hinblick auf die anfänglich vertretenen Ansichten (vgl. z.B. Heck 1931: 33ff.). In der jüngeren Vergangenheit ist eine (sehr knappe) Darstellung bei Sytsema (1998: 152ff.) vorzufinden. Die bisherigen Meinungsübersichten bieten weder eine Darstellung aller Meinungsrichtungen noch eine Zusammenstellung aller bisherigen Argumentationsstränge mit

1. Dieser Aufsatz ist aus einer Hausarbeit im Studiengang Frisistik an der Christian-Albrechts-Universität zu Kiel entstanden. Ich danke einem Gutachter von Us Wurk für nützliche Hinweise.

2. Die Formulierung ist an dieser Stelle bewusst offen gewählt, weil hierauf die spätere Herausarbeitung fußt, dass bisher nicht ausreichend zwischen dem Konzipieren als Rechtssatz und einem Verschriftlichen mündlicher Regelungen unterschieden wurde.

3. Popkema verwendet in diesem Zusammenhang den bezeichnenden Ausdruck ,bone of contention".

Us Wurk 68 (2019), s. 99-118; https://doi.org/10.21827/5d481192a6c78 
einer darauf aufbauenden Bewertung. Insbesondere der Meinungsstand nach Algra ist nicht in einer systematischen Zusammenstellung vorzufinden.

Ziel dieses Aufsatzes ist es, den aktuellen Meinungsstand darzustellen und die einzelnen Argumentationslinien nach Kategorien geordnet wiederzugeben sowie schließlich auf dieser Grundlage zu einer Bewertung zu kommen. Dabei wird versucht, nicht innerhalb der einzelnen Argumente zu einem Ergebnis zu kommen, sondern aus ihrer Gegenüberstellung und ihrem $\mathrm{Zu}$ sammenspiel mit den anderen vorgebrachten Argumentationssträngen Erkenntnisse zu gewinnen. Es folgt daher keine eingehende Untersuchung sämtlicher Detailfragen (und insbesondere keine Textanalyse ${ }^{4}$ ), sondern es verbleibt - zugunsten einer übergeordneten Bewertung - bei einer Übersicht der wesentlichen Züge. Auch ein Beleg eines bestimmten Ergebnisses wird nicht anvisiert.

Nach einer kurzen Übersicht über die Texte der 17 Küren und der 24 Landrechte (Ziffer 2.1) sowie der Hunsingoer Handschriften (Ziffer 2.2) wird zunächst der Meinungsstand dargestellt (Ziffer 3.1). Auf dieser Grundlage wird unter Ziffer 3.2 die Fragestellung erarbeitet. Unter Ziffer 3.3 werden die einzelnen Argumente im Hinblick auf die Frage der Sprache der erstmaligen Verschriftlichung nach Kategorien aufgeführt sowie nach Darstellung aller Argumentationslinien in einem gemeinsamen Abschnitt bewertet.

\section{Die 17 Küren, die 24 Landrechte und das Jus Vetus Frisicum}

\subsection{Texte}

Die 17 Küren und die 24 Landrechte stellen die wichtigsten gemeinfriesischen (,pan-Frisian“) Rechtsquellen dar (Bremmer 2009: 7). Sie sind nicht nur die frühesten, sondern auch die am weitesten verbreiteten altfriesischen Rechtstexte (Bremmer 2009: 9). Als ihr Geltungsgebiet wird das Westerlauwersche Friesland, Friesland zwischen Lauwers und Ems und Ost-Friesland angegeben (Sytsema 1998: 9). Eine weitere Besonderheit liegt darin, dass die 17 Küren und die 24 Landrechte in altfriesischen, lateinischen und niederdeutschen ${ }^{5}$ Versionen überliefert sind. Die in den Hunsingoer Handschriften enthaltenen lateinischen Versionen werden als „Jus Vetus Frisicum“ bezeichnet (Heck 1931: 33).

4. Zumal der Beweiswert einer Textanalyse für die hier bearbeitete Frage aufgrund der im weiteren Verlauf des Aufsatzes dargestellten Instabilität der Quellentexte als begrenzt erscheint.

5. Die niederdeutschen Fassungen werden im Folgenden nicht berücksichtigt, da sie unstreitig aus einer späteren Zeit stammen, vgl. z.B. von Richthofen 1888: 193. 
Der Textinhalt selbst ist älter als die erhaltenen Handschriften (Bremmer 2009: 9). Dabei sind die Küren älter als die Landrechte (Popkema 2014: 373). Darüber hinaus ist die Frage der genauen Entstehungszeit nicht geklärt (Popkema 2014: 373). Die meisten Autoren gehen von einer Entstehungszeit im 11. Jahrhundert aus (Sytsema 1998: 9). Bremmer (2009: 9) differenziert: Die Küren sollen aus dem späten 11. Jahrhundert stammen, während die Landrechte zwischen dem frühen 12. bis zur Mitte des 13. Jahrhunderts entstanden sein sollen (Bremmer 2009: 9). ${ }^{6}$

Auch innerhalb der einzelnen Texte weisen die Inhalte ein unterschiedliches Alter auf. Die 17 Küren sollen beispielsweise nicht als Ganzes verfasst worden, sondern im Laufe der Zeit aus vermutlich zehn Küren gewachsen sein (Algra 1998: 9f.). Einzelne Inhalte der Küren sollen bis ins 9. Jahrhundert zurückgehen (Algra 1998: 2).

\subsection{Handschriften}

Der erste bekannte friesische Rechtstext (auf Latein, „Lex Frisionum“) stammt ca. aus dem Jahr 800 und ist in einem Druck aus dem 16. Jahrhundert erhalten (Roeleveld 1990: 371). Erste Handschriften mit altfriesischem Inhalt, die noch erhalten sind, stammen von ungefähr 1200 (Bremmer 2009: $6)$.

Die 17 Küren und die 24 Landrechte sind in einer Vielzahl von Handschriften zu finden. Die einzelnen Versionen weichen jedoch deutlich voneinander ab (Bremmer 2009: 10). Die ältesten heute noch erhaltenen Aufzeichnungen dieser gemeinfriesischen Rechtstexte sind in der Rüstringer Handschrift R1 auf Altfriesisch zu finden (Popkema 2014: 374). Lateinische Versionen der gemeinfriesischen Texte sind in den Hunsingoer Handschriften ${ }^{7}$ (H1 und H2) enthalten (Popkema 2014: 374). Sie sind in mehreren Werken abgedruckt, beispielsweise bei von Richthofen (1880) und Hoekstra (1950).

Insgesamt sind vier lateinische Versionen erhalten, die allesamt aus dem östlichen Teil Frieslands stammen und wovon es sich bei denen aus H1 und H2 um die ältesten handelt (Popkema 2014: 374). Heute wird davon ausgegangen, dass es sich bei H1 um eine Abschrift von H2 handelt (Hoekstra 1950: 25f., Roeleveld 1990: 371). Beide werden im Tresoar (Frysk

6. Zur Frage der Datierung s. auch Popkema 2014: 373, Fußnote 20.

7. Die Hunsingoer Handschriften 1 und 2 werden nach ihrer Herkunft auch als ,Wicht ${ }^{`}$ und ,Scaliger' bezeichnet. Umfassende Informationen zur Herkunft der Handschriften gibt von Richthofen (1880: 63ff.). Detaillierte Ausführungen zu den Handschriften selbst sind bei Hoekstra (1950: 1ff.) zu finden. 
Histoarysk en Letterkundich Sintrum) in Leeuwarden (früher: Provinsjale Biblioteek fan Fryslân) aufbewahrt.

Es wird davon ausgegangen, dass zwei Vorlagen für $\mathrm{H} 2$ verwendet wurden: eine Zusammenstellung friesischer und lateinischer Texte und eine Handschrift mit den sog. Hunsingoer Privilegien (Roeleveld 1990: 371). Während die Hunsingoer Privilegien auf 1252 datieren, so dass das frühestmögliche Entstehungsdatum der Vorlage feststeht, stammt die andere Vorlage wohl frühestens aus der zweiten Hälfte des 12. Jahrhunderts, eher aus der ersten Hälfte des 13. Jahrhunderts (Hoekstra 1950: 43). Die Zusammenstellung der Handschrift $\mathrm{H} 2$ hat wahrscheinlich in der zweiten Hälfte des 13. Jahrhunderts stattgefunden (Roeleveld 1990: 371).

\section{Altfriesisch oder Latein?}

\subsection{Meinungsstand}

Ursprünglich hatte man angenommen, dass der Urtext auf Latein verfasst war (Algra 1991: 77ff., Fußnote 12). Zunächst hatte sich von Richthofen (1880: 20) für eine lateinische Urfassung ausgesprochen:

„Eine genaue Vergleichung der einzelnen Texte lässt mich nicht im Geringsten zweifeln, daß die Küren und Landrechte ursprünglich lateinisch aufgezeichnet wurden, daß man diesen lateinischen Urtext später in den einzelnen Landschaften ins Friesische übersetzte und dabei mehr oder weniger nach dem damals in der einzelnen Landschaft geltenden Recht abänderte und ergänzte."

Bereits kurz nach von Richthofen wurde jedoch von einer Vielzahl von Autoren die Priorität der altfriesischen Texte vertreten: Die Autoren Buitenrust Hettema (1892: 347), Kögel (1894: 242ff.), His (1899: 39ff.), Schröder/ Künßberg (1919: 734 Fußnote 6), Sievers (1919: 221) und im Hinblick auf die Landrechte auch $\operatorname{Siebs}^{8}$ (1902: 537) gingen - in unterschiedlichen Ausprägungen und mit unterschiedlicher Argumentation - davon aus, dass der Urtext auf Friesisch verfasst war (siehe die Darstellung bei Heck 1931: 33ff.).

Heck (1931: 46ff.; sowie aufbauend auf dessen Argumentation Krogmann/ Kersting 1972) ging von einer ursprünglich auf Latein erfolgten Verschriftlichung aus, wobei er diesen Text als Niederschrift eines altfriesischen Gesetzesvortrags wertete. Ihm schloss sich auch Markey (1981: 66f.) an.

8. Im Hinblick auf die Küren nimmt er jedoch einen lateinischen Urtext an, vgl. Siebs 1902: 537. 
Algra (1966: 88ff., 1991: 77ff.) war der Auffassung, dass der ursprüngliche Text auf Altfriesisch verfasst war und widerlegte ausführlich die Argumentation von Heck bzw. Krogmann/Kersting. Roeleveld (1990: 372) geht unter Verweis auf Algra davon aus, dass es mittlerweile herrschende Meinung sei, dass die lateinischen Texte aus dem Altfriesischen übersetzt wurden. Auch Sytsema (1998: 153) schließt sich ausdrücklich Algra an und nimmt an, dass der lateinische Text als Übersetzung aus dem Altfriesischen zu werten ist. Ebenso spricht sich Johnston (1998: 204) dafür aus, dass die lateinischen Texte aus dem Altfriesischen übersetzt sind. Popkema (2014: 386f.) vertritt ebenfalls die These der Priorität altfriesischer Versionen der 17 Küren und der 24 Landrechte.

Bremmer (2009:9) ist hingegen der Auffassung, dass sich ein wissenschaftlicher Konsens bisher noch nicht gebildet hat. Er spricht sich für einen lateinischen Ursprungstext aus und geht aber im Hinblick auf den konkreten lateinischen Text der Hunsingoer Version von einer Übersetzung aus dem Altfriesischen aus (Bremmer 2004: 110).

\subsection{Erstmalige Verschriftlichung vs. überlieferte Versionen}

$\mathrm{Zu}$ prüfen ist, ob mit Roeleveld davon auszugehen ist, dass der Meinungsstreit geklärt ist, oder mit Bremmer, dass die Frage nach wie vor offen ist. Bei einer systematischen Gegenüberstellung der einzelnen Positionen zeigt sich, dass eine Differenzierung zwischen der erstmaligen Verschriftlichung und den überlieferten Versionen vorzunehmen ist. Eine deutliche Differenzierung in dieser Hinsicht nimmt nur Bremmer (2004: 2010) vor. Vermutlich ist dies darauf zurückzuführen, dass er zu unterschiedlichen Ergebnissen kommt, nämlich zu einer erstmaligen Verschriftlichung auf Latein, obwohl er den lateinischen Hunsingoer Text als aus dem Altfriesischen übersetzt ansieht, während die anderen Autoren bei beiden Fragen zu dem Ergebnis einer altfriesischen Sprachpriorität kommen.

Unklar ist nämlich bei der bisherigen Behandlung der Thematik häufig geblieben, welche Fragestellung zugrundegelegt wird. Daher werden im Folgenden die zentralen vertretenen Thesen aufgeführt:

(1) Ursprünglich wurden die betreffenden Rechtstexte mündlich überliefert.

(2) Bei dem in $\mathrm{H} 1 / \mathrm{H} 2$ überlieferten lateinischen Text handelt es sich nicht um die erstmalige Verschriftlichung.

(3) Bei dem in $\mathrm{H} 1 / \mathrm{H} 2$ überlieferten altfriesischen Text handelt es sich nicht um die erstmalige Verschriftlichung.

(4) Der in $\mathrm{H} 1 / \mathrm{H} 2$ überlieferte lateinische Text ist eine Übersetzung aus dem Altfriesischen. 
(5) Die Sprache der erstmaligen Verschriftlichung war Altfriesisch/ Latein (ungeklärt).

Aus einem Vergleich der in der Literatur geäußerten Positionen zeigt sich, dass in überwiegender Hinsicht keine Widersprüche vorliegen:

$\mathrm{Zu}$ (1): Es ist völlig unumstritten geblieben, dass ursprünglich eine mündliche Rechtstradition in altfriesischer Sprache bestand (vgl. z.B. Markey 1981: 66, Roeleveld 1990: 373, Bremmer 2014: 1ff.). Lediglich im Hinblick auf die Ausprägung dieser mündlichen Tradition und die angeführte Argumentation sind Unterschiede zwischen den Autoren zu erkennen. Die ursprüngliche, im 19. Jahrhundert entwickelte Argumentation fußt auf der Bezeichnung des Asega mit der wörtlichen Bedeutung Gesetzessprecher (Popkema 2014: 380, Fußnote 40). Der Asega auf dem Upstalsboom wurde vielmals verglichen (und gleichgestellt, vgl. Markey 1981: 66, 103) mit dem isländischen logsogumaðr auf dem Thingvellir. Bereits Heck (1931: 38f.) hatte sich gegen diese Einstufung des Asega gewandt und wertete ihn auf der Basis der überlieferten Texte als „Urteilfinder“. ${ }^{9}$ Auch Bremmer (2014: 1f.) widerspricht dem Verständnis des Asega als Gesetzessprecher unter Verweis auf fehlende Belege in den Quellen. Er geht davon aus, dass der Asega nicht Gesetze rezitierte, sondern bei Versammlungen anwesend war, sich das Vorgetragene anhörte und dann auf der Grundlage seiner Rechtskenntnis ein Urteil traf (Bremmer 2014: 30f.).

Vielfach wurden Alliteration und Rhythmus als dichterische Elemente angeführt und ein Vergleich mit der nordischen Tradition (Eingangsformel, Metrik) vorgenommen (Heck 1931: 40f.). Bremmer (2014: 39) wertet diese Elemente nicht als eine als Erinnerungsstütze innerhalb einer mündlichen Tradition eingesetzten Dichtkunst, sondern schlicht als funktionale Merkmale der Mündlichkeit der Situation (,functional features of orality").

$\mathrm{Zu}$ (2): Weiterhin herrscht bei allen Autoren Einigkeit darüber, dass es sich bei dem lateinischen Text aus $\mathrm{H} 1 / \mathrm{H} 2$ jedenfalls nicht um die erstmalige Verschriftlichung handelt. Dies vertreten nicht einmal Heck und von Richthofen. Beide gestehen ein, dass es sich bei dem überlieferten lateinischen Text nicht um die allererste Niederschrift gehandelt hat: Von Richthofen (1880: 21, 193), der das Jus Vetus Frisicum für eine Wiedergabe des ursprünglichen Textes hält, wertet den auf Latein überlieferten Text eben lediglich als eine Wiedergabe und weist auf vorgenommene Kürzungen und

9. Er geht allerdings davon aus, dass es einen Gesetzesvortrag durchaus gab (nur nicht durch den Asega), und baut darauf seine gesamte Argumentation in der Frage der Sprachpriorität auf (vgl. Heck 1931: 39ff.). 
Änderungen hin. Auch Heck (1931: 79) bewertet den Lateintext als Überlieferung. ${ }^{10}$

$\mathrm{Zu}$ (3): Die altfriesischen Versionen in $\mathrm{H} 1$ und $\mathrm{H} 2$ sind unstreitig erst nach einer erstmaligen Verschriftlichung verfasst worden. Es wird davon ausgegangen, dass die Rechtstexte vor ihrem Erscheinen in der ältesten überlieferten Handschrift zumindest in Teilen eine gewisse Zeit sowohl in mündlicher als auch in schriftlicher Form existierten (Bremmer 2004: 113).

$\mathrm{Zu}$ (4): Die lateinischen Versionen in $\mathrm{H} 1$ und $\mathrm{H} 2$ (das sog. Jus Vetus Frisicum) stehen - heutzutage ebenfalls unstreitig - höchstens neben den altfriesischen Versionen, da davon ausgegangen wird, dass sie auf friesischen Texten ${ }^{11}$ beruhen (vgl. z.B. Algra 1966: 88ff.). Hierauf bezieht sich Roeleveld (1990: 372) mit ihrer Aussage, die Frage sei geklärt.

Eine andere Auffassung vertraten Heck und von Richthofen: Von Richthofen (1880: 193) hielt die friesischen Texte für „Überarbeitungen“ (also Übersetzungen) des Jus Vetus Frisicum, also gerade der lateinischen Version der Hunsingoer Handschriften. Auch Heck (1931: 35ff.) sprach sich klar für eine (Rück-)Übersetzung der altfriesischen Texte aus dem überlieferten Jus Vetus Frisicum aus, welches er als Niederschrift eines mündlichen Gesetzesvortrags zu Protokoll ansah.

Diese These wurde, wie oben bereits erwähnt, von mehreren Autoren noch Ende des 19. und Anfang des 20. Jahrhunderts in Frage gestellt. ${ }^{12}$ Bis heute ist die Ansicht vorherrschend geblieben, dass das in $\mathrm{H} 1$ und $\mathrm{H} 2$ überlieferte Jus Vetus Frisicum aus dem Altfriesischen übersetzt wurde (Algra 1991: 82; Roeleveld 1990: 372). Als Begründung wird auf die enthaltenen altfriesischen Begriffe $^{13}$ sowie die Verwendung einzelner Begriffe verwiesen, die in einem lateinischen Text als fehlerhaft anzusehen sind (Sytsema 1998: 153).

10. Er schreibt wörtlich: „Haben wir eine treue Überlieferung der ursprünglichen amtlichen Niederschrift vor uns, oder eine Bearbeitung? Die Frage ist in der Hauptsache im ersteren Sinn zu beantworten. Der ursprüngliche Text hat sicher Zusätze und Einschiebungen erfahren [...]. Auch fehlt es nicht ganz an auf Abschrift zurückgehenden Korruptelen. [...] Auch die Zusätze halten sich in sehr bescheidenen Grenzen. Eine Umarbeitung hat nicht stattgefunden. Sie würde gewisse Fehler sicher beseitigt haben. Was uns vorliegt ist $\mathrm{m}$. E. der fast unveränderte Text des ursprünglichen, offiziellen Rechtsprotokolls.“ (Hervorhebungen durch die Verfasserin).

11. Dies meint gerade nicht zwingend, dass sie aus den altfriesischen Versionen der Hunsingoer Handschriften übersetzt wurden. Es kommen auch andere Vorlagen in Betracht.

12. Siehe auch die Darstellung bei Algra (1991: 77ff.) sowie die Übersicht der bis 1931 vertretenen Thesen bei Heck (1931: 34f.).

13. Vgl. die Liste mit Erläuterungen bei Roeleveld (1990: 376ff.). 
$\mathrm{Zu}$ (5): $\mathrm{Zu}$ einem bestimmten Zeitpunkt kam es zu einer erstmaligen Verschriftlichung des zuvor mündlich überlieferten Rechts. An diesem Punkt stellt sich die - nach wie vor ungeklärte - Frage, ob diese erste Niederschrift auf Latein oder auf Altfriesisch vorgenommen wurde. Die Aussagen, die Priorität der altfriesischen Texte sei geklärt, beziehen sich ausschließlich auf die konkrete lateinische Version der Hunsingoer Handschriften. ${ }^{14}$

Die Sprache der ersten Niederschrift ist insbesondere vor dem Hintergrund von Bedeutung, dass sie Auswirkungen auf den Inhalt haben kann. Die Verwendung von Latein in einem rechtlichen Kontext bringt eine bestimmte, feste Bedeutung der verwendeten Terminologie mit sich, die nur innerhalb eines bestimmten Rechtssystems funktioniert und deren Nutzung außerhalb dieses Systems zu massiven Bedeutungsänderungen führen kann (Algra 1998: 32). Die Reihenfolge der Übersetzung spielt ebenfalls eine Rolle, da jede Übersetzung dazu führt, dass eine Abweichung vom ursprünglichen Inhalt vorliegt (Algra 1998: 28f.).

Im Hinblick auf die Argumentation zu Ziffer (5) wurden in der Vergangenheit unterschiedliche Linien verfolgt. Lange Zeit lag der Fokus der Diskussion auf textinternen Argumenten. Da diese die Frage der Sprachpriorität nicht abschließend klären konnten, schlägt Popkema (2014: 370) eine Orientierung über das Textargument hinaus hin zu neuen Perspektiven, insbesondere unter Einbeziehung soziokultureller Aspekte, vor. Auch diesem Artikel liegt die Idee zugrunde, dass eine Erweiterung des Fokus erforderlich ist und dass insbesondere eine Kombination verschiedener Argumentationsstränge die besten Erfolgschancen verspricht. Im Folgenden werden daher die unterschiedlichen Linien dargestellt.

\subsection{Die Sprache der erstmaligen Verschriftlichung}

\subsubsection{Der Übergang von mündlicher zu schriftlicher Tradition}

Wie bereits dargestellt, bestand zunächst eine Rechtstradition in mündlicher Überlieferung auf Altfriesisch. Im Hinblick auf die Sprache der erstmaligen Verschriftlichung stellt sich zunächst die Frage, wie der Übergang zu einer schriftlichen Tradition erfolgt ist. Dabei ist zu berücksichtigen, dass bei dem Übergang zu einer schriftlichen Tradition von der Anwendung eines „Filters“

14. So schreibt Roeleveld (1990: 372): ,[...] and in no cases were Old Frisian versions based on this Latin version [...]“; auch Sytsemas Meinung (1998: 153) bezieht sich ausdrücklich auf die konkreten lateinischen Texte der Handschrift (,[...] dat $\underline{\mathrm{L}}$ als vertaling uit het Oudfries beschouwd moet worden" (Hervorhebungen durch die Verfasserin). 
ausgegangen werden muss: „Rather than being a straight record of oral dictation, however, most of these written laws and related texts must already have passed a filter of literacy." (Bremmer 2014: 5). Des Weiteren ist davon auszugehen, dass es sich um einen graduellen Übergang handelte, also für eine gewisse Zeit mündliche und schriftliche Tradition parallel bestanden und sich gegenseitig beeinflussten (Bremmer 2014: 4). Die ersten Texte sind folglich als eine Art Zwischenstufe anzusehen. Darüber hinaus ist davon auszugehen, dass die Form der Sprache im Rahmen des komplizierten Vervielfältigungsprozesses aktualisiert wurde (Bremmer 2009: 12). Auf die in dieser Hinsicht bestehende „Offenheit" (,openheid“) der Texte der 17 Küren und der 24 Landrechte und eine daraus resultierende Instabilität weist Bremmer (2004: 111) ausführlich hin.

Der Grund für die Entstehung einer schriftlichen Sammlung der Rechtstexte wird hauptsächlich in einem privaten Interesse zur praktischen Verwendung gesehen (Bremmer 2009: 13; jedoch Heck 1931: 36, 76ff.: zur amtlich veranlassten Protokollierung). Johnston (1998: 212) hält dies grundsätzlich zwar für korrekt, weist jedoch im Hinblick auf die 17 Küren und die 24 Landrechte auf einen ,unverkennbaren ideologischen Charakter“ (,unmistakably ideological character") hin. Dieser kann aber auch, wie er selbst schreibt (1998: 212), bei einer späteren Zusammenstellung der einzelnen Texte entstanden sein, so dass ursprünglich durchaus private Texte (,older, initially informal collections") als Vorlage gedient haben können. Ideologische Hintergründe werden über die einzelnen Texte hinaus aber insbesondere auf Ebene der Sammlungen eine Rolle gespielt haben. Ziel war vor allem, die friesische Unabhängigkeit im Heiligen Römischen Reich zu stützen (Bremmer 2009: 13).

\subsubsection{Entwicklung der Rechtssätze}

Der mündliche Ursprung sowie der private Charakter der Aufzeichnungen spielen auch in einem anderen Zusammenhang eine wichtige Rolle: Bei den Sammlungen der friesischen Rechtstexte handelt es sich gerade nicht um von einem Gesetzgeber verabschiedete Gesetze (Bremmer 2009: 13).

Lediglich Heck (1931: 78) hatte vertreten, dass es sich bei der erstmaligen Verschriftlichung der 17 Küren und der 24 Landrechte auf Latein um eine „Übersetzung auf offizielle Veranlassung bei Genehmigung der Rechtssatzungen durch eine normgebende Versammlung“" gehandelt habe. Diese rechtshistorische These ist ein wichtiger Bestandteil seiner Argumentation, findet jedoch keine Unterstützung in den Aussagen der anderen Autoren zum Entstehungsprozess des altfriesischen Rechts. 
Für den Prozess der Entwicklung der Rechtssätze wird zwischen den Küren und den Landrechten unterschieden. Das altfriesische Recht bestand im Wesentlichen aus Rechtsprechung, wobei jedes neue Urteil das bestehende Recht erweiterte (Bremmer (2014: 24). Speziell im Hinblick auf die 24 Landrechte wird davon ausgegangen, dass sie auf Rechtsprechung zurückgehen (Algra 1991: 349). Es erfolgte also keine geplante und umfassende abstrakte Formulierung, sondern dieser Rechtstext bestand aus einer Vielzahl an konkret entschiedenen Einzelfällen.

Bei den Küren handelt es sich um eine andere Art der Rechtsquelle. Für sie wird angenommen, dass es einen Gesetzentwurf gab, der in einer Volksversammlung akklamiert wurde (Algra 2001: 556f.). Dem folgte noch ein Erlass durch den Landesherrn (Algra 2001: 557).

\subsubsection{Latein als offizielle Verwaltungssprache in Westeuropa}

Eine besondere Stellung in der Diskussion um die Sprachpriorität nimmt der geschichtliche Hintergrund der lateinischen Sprache als zur damaligen Zeit in Westeuropa klar dominierenden Verwaltungssprache ein. Latein war vor allem die Sprache der Kirche und des Kirchenrechts (Roeleveld 1990: 373). Zudem war es die Sprache des Römischen Rechts, welches das friesische Recht früh beeinflusste, da ab 1200 friesische Juristen in Römischem und Kirchenrecht u.a. in Frankreich ausgebildet wurden (Bremmer 2004: 97f., Roeleveld 1990: 373).

Bedeutsam für die Zeit der Verschriftlichung der 17 Küren und der 24 Landrechte ist, dass in der Umgebung Frieslands Kodifizierungsprozesse zunächst üblicherweise auf Latein stattfanden (Popkema 2014: 380). Auch darüber hinaus war Latein bis ins Hochmittelalter die Sprache, die in einem schriftlichen Kontext und insbesondere für Rechtstexte verwendet wurde (Popkema 2014: 380). Zu einem Übergang zu einer Verwendung der jeweiligen Volkssprachen in einem rechtlichen Zusammenhang kam es erst später, im Verlauf des 12. und 13. Jahrhunderts (Popkema 2014: 380). Dennoch wurden bis ins vierzehnte Jahrhundert hinein friesische Dokumente auf Latein verfasst (von Richthofen 1888: 99).

In den friesischen Gebieten bestand jedoch eine im Vergleich zur westeuropäischen Umgebung besondere Situation. Popkema (2014: 371) geht daher davon aus, dass sich die Situation in Friesland anders entwickelte als im restlichen Westeuropa. Ab 793 waren die Friesen Teil des Fränkischen Reichs (Bremmer 2014: 3). Allerdings waren die Franken dort u.a. wegen der geographischen Lage mit einer Marschlandschaft sowie wegen wiederkehrender Einfälle der Wikinger zu keinem Zeitpunkt tatsächlich präsent, was 
dazu führte, dass das Gebiet im Laufe der Zeit seine Unabhängigkeit (Johnston 1998: 179: ,a sort of de facto independence") zurückerhielt und es ab ca. 1100 östlich der Lauwers und ab ca. 1250 westlich der Lauwers in Selbstverwaltung stand (Bremmer 2014: 3f.). Die Zugehörigkeit zum Heiligen Römischen Reich war schließlich nur formal (Popkema 2014: 382). Anstelle mit Holland und Sachsen ist daher eher ein Vergleich mit der Entwicklung in England, Island und Skandinavien anzustellen, wo die ältesten Rechtstexte ausnahmslos in der Volkssprache und nicht auf Latein überliefert sind (Popkema 2014: 381ff.).

\subsubsection{Außendarstellung und Ideologie}

Für das Erstellen einer lateinischen Version ${ }^{15}$ werden Gründe der Außendarstellung oder ideologische Gründe vorgebracht. Möglicherweise sollten Leser außerhalb Frieslands als Zielgruppe angesprochen werden (Bremmer 2004: 118) oder der Kontakt zu Nicht-Friesisch-Sprechern könnte ein Grund für eine lateinische Version gewesen sein (Roeleveld 1990: 373). Eine lateinische Version könnte möglicherweise wichtig für Lehnsherren von außerhalb Frieslands gewesen sein (Roeleveld 1990: 374). Johnston (1998: 204) hingegen sieht die lateinischen Texte in $\mathrm{H} 1$ und $\mathrm{H} 2$ als , [...] nothing less than an ideological brochure containing documents legitimizing the Frisians' claim to independence, carefully selected for export to the skeptical outside world." Die Erwähnung von Textelementen zur Freiheit der Friesen sollte die politische Sonderstellung der friesischen Gebiete gegenüber fremden Mächten legitimieren (Johnston 1998: 180). Markey (1981: 102) sieht ebenfalls mit der Anführung der Freiheit der Friesen einen ideologischen Zweck in der Verschriftlichung der 17 Küren und der 24 Landrechte und verweist auf die Notwendigkeit dieser Bestätigung im 11. und Anfang des 12. Jahrhunderts wegen der Teilung Frieslands durch den Kaiser und den daraus resultierenden gewalttätigen Ausschreitungen. Auch Algra (1991: 67f., Fußnote 43) geht davon aus, dass es darum ging, die Sonderstellung Frieslands zu rechtfertigen, und nennt als konkreten Anlass die mögliche Verwendung der Texte als Argument im Streit der Ommelande mit der Stadt Groningen im 13. Jahrhundert.

\section{*3.3.5 Textbestand als Argument}

Eine neuere Argumentation nimmt den Textbestand in den Blick. Popkema (2014: 372ff.) erläutert, dass der erhaltene Textbestand im Hinblick auf die

15. Allerdings sind sie auch nur dies: Gründe für die Erstellung einer lateinischen Version. Sie liefern keinen Grund für eine erstmalige Verschriftlichung auf Latein. Vgl. hierzu auch unten unter 3.3.7. 
Schrifttradition, insbesondere auf das Verhältnis zwischen den Schriftsprachen, auf friesischem Gebiet als repräsentativ angesehen, und er daher als Indikator für die Sprachpriorität herangezogen werden kann. Weiter argumentiert er mit einer Unterscheidung zwischen zwei Schrifttraditionen: Er nimmt an, dass der Originaltext zunächst in der Sprache des Originals kopiert wurde, woraus ein Korpus und damit eine Tradition entstand, die er als „primary written language tradition“ bezeichnet. Mit der Übersetzung des Texts und den in der Folge angefertigten Kopien entstand dann eine „secondary written language tradition“ (Popkema 2014: 375ff.). In einem weiteren Schritt argumentiert er, dass, wenn ein Text nur in einer Sprache überliefert ist, dies dafür spricht, dass es sich bei dieser um die Sprache der primary written language tradition handelt, obwohl er dieser These einen gewissen Zufallsfaktor zugesteht (Popkema 2014: 377). Auf dieser Grundlage stellt er die These auf, dass die stärkere Tradition als die primary written language tradition und die schwächere als die secondary written language tradition anzusehen ist (Popkema 2014: 378).

Bei den Texten, für die auch eine lateinische Version erhalten ist, ist die älteste erhaltene Version in allen Fällen auf Altfriesisch (Popkema 2014: 375). Außerdem ist diese altfriesische Texttradition stärker als die lateinische, mit Ausnahme der Upstalsboom-Gesetze (Popkema 2014: 375f.).

In der Tat ist vor diesem Hintergrund nur schwerlich zu erklären, wie es im Fall einer Priorität lateinischer Texte dazu gekommen sein soll, dass hauptsächlich altfriesische Versionen überliefert wurden, während keine oder kaum lateinische Versionen erhalten sind (vgl. Popkema 2014: 378).

Darüber hinaus zeigt Popkema (2014: 378f.) eine Trennung nach Domänen auf. Damit erklärt er, warum kein Widerspruch zur Annahme einer erstmaligen Verschriftlichung von Rechtstexten auf Altfriesisch bestehen muss, obwohl bei manchen Texten durchaus eine starke lateinische Tradition vorzufinden ist: Nur die friesischen Rechtstexte sind deutlich überwiegend auf Altfriesisch erhalten, während fast alle anderen Textsorten auf Latein erhalten sind. Bei diesen anderen Textsorten handelt es sich beispielsweise um religiöse Texte oder Römisches oder Kirchenrecht bzw. dessen Kommentierung (Popkema 2014: 378).

\subsubsection{Textbasierte Argumentation}

Die bisherigen Untersuchungen zur Frage der Sprache der erstmaligen Verschriftlichung konzentrierten sich überwiegend, wenn nicht sogar ausschließlich auf Argumentationslinien auf der Grundlage des Textes selbst (Popkema 2014: 369f.). 
Zur Klärung der Sprachpriorität kann nicht auf Metakommentare im Text zurückgegriffen werden, denn anders als z.B. im Sachsenspiegel, in dem eine lateinische Urversion ausdrücklich im Prolog erwähnt wird, weisen die betreffenden Rechtsquellen derartige Kommentare nicht auf (Popkema 2014: 377, Fußnote 29). Für die Frage nach der Sprache der erstmaligen Verschriftlichung wurde der Schwerpunkt der Textuntersuchung bisher vor allem auf offensichtliche Fehler des Lateintextes gelegt. Heck (1931: 46) vertritt unter Hinweis auf die dort enthaltenen gravierenden sprachlichen Fehler, dass es sich bei der lateinischen Version um die erste Verschriftlichung eines mündlichen, altfriesischen Gesetzesvortrags gehandelt haben muss, da derartige Fehler ,[...] bei einer Arbeit in der Studierstube nach schriftlicher Vorlage vermieden worden [wären]“. Seiner Bewertung schließt sich Markey (1981: 66f.) an. Im Zentrum von Hecks Argumentation steht das Durchschlagen von Fehlern im lateinischen Text auf den altfriesischen Text:

„Wenn eine Korruptel, die nur bei der Anfertigung des Lateintextes entstanden sein kann, sich in allen friesischen Texten wiederfindet, dann wird gleichsam das Problem der Filiation durch den Nachweis einer erblichen Belastung gelöst.“ (Heck 1931: 48)

Seine Argumentation wurde jedoch durch Algra (1966: 92ff., 1991: 95ff.) ausführlich und überzeugend widerlegt. Es soll hier bei dem Verweis auf die erheblichen methodischen Fehler Hecks verbleiben, die Algra anführt:

„Hem moet met name verweten worden, dat hij de meningen van anderen en het bronnenmateriaal zeer oppervlakkig bestudeerde. [...] Methodisch lijkt mij ontoelaatbaar dat hij werkt met door hem zelf bedachte Oudfriese woorden. [...]“" (Algra 1991: 120)

Bremmer (2004: 114ff.) erweitert die Textargumentation auf den Prolog der 17 Küren und der 24 Landrechte und kommt zu dem Ergebnis eines lateinischen Urtextes. Dabei stützt er sich neben weiteren Aspekten insbesondere auf im Text enthaltene lateinische Wendungen (Bremmer 2004: 115f.). Er hält es für unwahrscheinlich, dass willkürlich lateinische Zusätze in einen altfriesischen Originaltext eingestreut wurden, und wertet diese daher als Zeichen einer Übersetzung aus einem ursprünglich lateinischen Text (Bremmer 2004: 116). Seine Argumentation hält Popkema für nicht gänzlich überzeugend, da vielfältige Interpretationen der von Bremmer angeführten Textbeispiele denkbar seien (zur vielfältigen und umfangreichen Argumentation siehe Popkema (2014: 371, 2005: 107 ff.)). 


\subsubsection{Bewertung}

Die Annahmen zum Übergang von mündlicher zu schriftlicher Tradition geben bereits deutliche Hinweise im Hinblick auf die Frage der Sprachpriorität: Die ersten Texte waren keine „fertigen“, festen Texte. Sie wurden ständig sprachlich aktualisiert, aber auch verändert. Hinzu kam, dass mündliche und schriftliche Tradition eine Zeit lang parallel existiert haben müssen.

Insbesondere die hierbei angenommene, stetig neue gegenseitige Beeinflussung spricht für ein Nebeneinanderbestehen einer altfriesischen mündlichen Tradition mit einer altfriesischen schriftlichen Tradition. Da der inhaltliche Ursprung des Rechts in der mündlichen Rechtstradition und damit auf Altfriesisch zu sehen ist, muss es, um zu einer schriftlichen Version auf Latein zu kommen, zu irgendeinem Zeitpunkt zu einer Übersetzung gekommen sein. Es ist unwahrscheinlich, dass bei jeder Änderung oder Überarbeitung in der mündlichen Sphäre eine schriftliche Umsetzung auf Latein erfolgte, da es wesentlich einfacher und praktischer ist, nur in einer Sprache zu arbeiten, als jeden einzelnen Schritt in eine andere Sprache zu übersetzen - und dann bei Anwendung des Textes in einem bestimmten Rechtszusammenhang wieder zurück in die Ausgangssprache. ${ }^{16}$

Hinzu kommt, dass den Rechtstexten kein Gesetzgebungsverfahren in einem modernen Sinn zugrunde lag. Eine Konzeption der Rechtssätze in Textform, wie man es sich heutzutage vorstellt, hat nicht stattgefunden. Es ist wichtig, die von unserer heutigen Vorstellung abweichende Rechtsrealität in die vorliegende Diskussion miteinzubeziehen, und kann daher irreführend sein, im Hinblick auf die Sprache der erstmaligen Verschriftlichung von einem Entwerfen von Rechtstexten zu sprechen. ${ }^{17}$

Das Recht galt nicht erst durch eine Niederschrift (oder gar eine Veröffentlichung des schriftlichen Textes), sondern unabhängig davon; es war zuvor mündlich überliefert worden und bereits seit längerer Zeit wirksam. Es handelte sich also bei der Verschriftlichung der Rechtstexte um ein „Aufschreiben“" von etwas, das bereits bestand. Da dieses „Aufschreiben“ keinen modernen rechtsstaatlichen Grundsätzen, z.B. dem Bestimmtheitsgrundsatz,

16. Anders wäre dies jedoch, wenn man davon ausginge, dass schlicht immer, aus Prinzip, ausschließlich auf Latein geschrieben geworden wäre (siehe hierzu die folgenden Ausführungen zu Latein als etablierter Schriftsprache). Dies müsste aber auch zu einer hohen Zahl an entsprechenden lateinischen Versionen geführt haben, was zwar nicht ausgeschlossen ist, im erhaltenen Textbestand jedoch keine Stütze findet (zum Textbestand s.o. Ziffer 3.3.5).

17. Popkema verwendet beispielsweise Ausdrücke wie „drafting Frisian legal texts” (2014: 378). 
gerecht werden musste, und es auch keine (ändernde) Wirkung auf das geltende Recht hatte, ist davon auszugehen, dass die ersten Texte vergleichsweise instabil waren. ${ }^{18}$ Ebenso gab es aufgrund dieser fehlenden Bindung an einen festen Wortlaut nicht nur eine einzige Fassung, der man Gültigkeit zusprach, sondern eine Vielzahl an voneinander abweichenden Versionen. Gerade die Festlegung auf einen genauen Wortlaut war nicht Ziel der Verschriftlichung, wie bereits die inhaltlichen Unterschiede zwischen den erhaltenen Versionen der 24 Landrechte und 17 Küren zeigen.

Des Weiteren spielt eine Rolle, dass die Küren und Landrechte nicht in einem einzigen Gesetzgebungsakt und damit zu einem einzigen Zeitpunkt aufgestellt wurden. Die Landrechte entwickelten sich aus einer Vielzahl an einzelnen entschiedenen Rechtsfällen. Aber auch bei den Küren wird davon ausgegangen, dass sie zunächst in einer geringeren Anzahl vorlagen und im Laufe der Zeit ergänzt wurden. Insofern ist sowohl ein Zeit- als auch ein Entwicklungsfaktor zu berücksichtigen.

Hinzu kam die praktische Problematik der Kopierfehler, die zu Änderungen des Textes führten. Auch unter diesen Aspekten waren die Texte nicht „fest“. Textbasierten Argumenten kann aufgrund der fehlenden Absolutheit der Texte kein hoher Beweiswert zugemessen werden.

Wenn es sich lediglich um ein „Aufschreiben“ ohne offiziellen Charakter handelte, erklärt sich nicht, warum man in einem rein friesischen Umfeld Rechtstexte auf Latein verschriftlichen sollte. Die in diesem Zusammenhang häufig angeführte Dominanz der lateinischen Sprache als offizielle Verwaltungssprache in Westeuropa hat nach dem bisherigen Kenntnisstand für die friesischen Gebiete in dieser Ausprägung nicht bestanden. Die unabhängige Sonderstellung Frieslands ist offensichtlich, so dass der von mehreren Autoren vorgenommene Vergleich mit der Entwicklung in den nordischen Ländern, die ihre Rechtstexte unmittelbar in den Volkssprachen verschriftlichten, naheliegend ist. Auch unter diesem Aspekt ist folglich eine erstmalige Verschriftlichung der Rechtstexte auf Altfriesisch wahrscheinlich.

In ideologischer Hinsicht ist der Gedanke überzeugend, dass im Verhältnis nach außen eine Präsentation der Texte auf Latein sinnvoll war. Dies bedeutet jedoch nicht, dass die erstmalige Verschriftlichung zwingend auf Latein erfolgt sein muss, sondern eher, dass es die Texte zumindest auch in einer lateinischen Version gab. Maßgeblich bei dieser Frage ist der Grund der

18. Zu diesem Ergebnis kommt auch Bremmer (2004: 111) auf der Grundlage der großen Vielfalt der einzelnen Texte. 
Verschriftlichung des zuvor mündlich überlieferten Rechts. Am Naheliegendsten sind private, jedenfalls ,innerfriesische“ Gründe, insbesondere der Dokumentation und der erleichterten Arbeit. Bei einer Verschriftlichung zu privaten Zwecken scheint der Gedanke, etwas für sich selbst und zur weiteren Bearbeitung in einer fremden Sprache aufzuschreiben, aber unwahrscheinlich. Sinnvoller scheint das Erstellen einer lateinischen Version daher erst in einem zweiten Schritt: Man schrieb die mündlichen Rechtssätze in der Sprache auf, in der sie gesprochen wurden, also auf Altfriesisch, und nutzte diese Version anschließend zum Zweck des Transports ideologischer Inhalte nach außen, indem man auch eine lateinische Version erstellte.

Wenn man die Texte nur zur Außendarstellung aufgeschrieben hätte (was aufgrund des hier angenommenen und zumeist vertretenen privaten, praktischen Charakters unwahrscheinlich scheint) und aus diesem Grund Latein verwendete, stellt sich die Frage, warum so viele altfriesische und kaum oder keine lateinischen Fassungen existieren. Der von Popkema untersuchte Textbestand ist ein starkes Argument. Auch seine Trennung nach Domänen vermag die Unterschiede bei der Sprachverwendung sehr gut zu erklären.

Das einzige Problem an dieser Argumentation ist die geringe Zahlenbasis, auf der Popkemas These aufbaut. Der erhaltene Bestand ist sehr klein, so dass es schwierig ist, hieraus eine absolute Aussage zu gewinnen. Dies versucht er jedoch auch gar nicht, sondern es geht ihm nur um eine Indizwirkung. Das stärkste Argument für eine Priorität der altfriesischen Texte ist dabei gar nicht die stärkere Tradition der altfriesischen Texte, sondern vielmehr die überraschend geringe Anzahl erhaltener lateinischer Fassungen, für die es, sollte man sie als ,primary written language tradition“ ansehen wollen, keine Erklärung gibt. Popkema liefert mit seinem Ansatz und der Einstufung der lateinischen Texte als „secondary written language tradition“ eine plausible Erklärung für das Verhältnis der Sprachen zueinander im Textbestand.

$\mathrm{Zu}$ Argumenten, die sich direkt aus den einzelnen Texten ergeben, hat es von Beginn der Diskussion der Frage an umfangreiche Untersuchungen mit zahlreichen Vergleichen der Texte untereinander gegeben. Heck hat mit seinem Gedankengang der ,erblichen Belastung“ zwar einen guten Ansatzpunkt gewählt, jedoch nicht berücksichtigt, dass es sich bei seiner Prüfgrundlage nicht um den Urtext handelt. Dies war ihm zwar bewusst (vgl. oben Fußnote 9), er ging jedoch dennoch von einer ,treuen Überlieferung“ aus. Diese Bewertung berücksichtigt mögliche Veränderungen nicht in ausreichendem Maße. Bis zum Entstehen dieser Handschriften muss eine gewisse Zeit vergangen sein, in der ihre Vorlagen (bzw. evtl. sogar mehrere Generationen solcher Vorlagen) bereits in Umlauf waren, und für die davon ausgegangen 
werden muss, dass eine Vielzahl von Änderungen (seien es bewusste Abänderungen oder lediglich Kopierfehler) ihren Weg in $\mathrm{H} 1 / \mathrm{H} 2$ gefunden haben. Damit bleibt völlig unklar, welche Rolle die Vorlagen, die für $\mathrm{H} 1 / \mathrm{H} 2$ verwendet wurden, bei den von Heck als Argumentation herangezogenen Fehlern spielten. Vor diesem Hintergrund kann eine klare Entwicklungsreihenfolge, wie er sie zu etablieren versucht, nicht rekonstruiert werden, zumal die Texte womöglich auf der Grundlage mehrerer, sich vermutlich sogar widersprechender Vorlagen und/oder durch mehrere Verfasser erstellt wurden.

$\mathrm{Zu}$ in der lateinischen Fassung enthaltenen altfriesischen Wörtern bleibt festzuhalten, dass ihr Beweiswert nicht überbewertet werden sollte. Da alle Autoren unstreitig davon ausgehen, dass ursprünglich ein mündlicher altfriesischer Inhalt existierte, überrascht es nicht, dass altfriesische Begriffe in einer lateinischen Fassung vorhanden sind. Ihre Existenz ist sowohl im Rahmen einer erstmaligen Niederschrift auf Latein als auch bei einer Übersetzung aus einer schriftlichen altfriesischen Vorlage denkbar. Insofern scheint eine auf diesem Aspekt aufbauende Beweiskette jedenfalls nicht als ausreichend für eine eindeutige Bestimmung der Sprache der ersten Niederschrift.

Auch für die lateinischen Wendungen im Prolog kann es mehrere sinnvolle Begründungen geben (z.B. die Verwendung lateinischer Floskeln aus stilistischen Gründen), so dass auch hier der Beweiswert nicht als besonders hoch einzustufen ist.

\section{Zusammenfassung und Ausblick}

Im Hinblick auf die Frage der Übersetzungsreihenfolge wurde zunächst herausgearbeitet, dass bei einer genauen Prüfung der einzelnen vertretenen Thesen kaum Widersprüche verbleiben. Lediglich die Ausprägungen und Begründungen variieren. Als einzige offene Frage stellt sich die Frage nach der Sprache der allerersten Verschriftlichung heraus. Nach einer Darstellung der vertretenen Argumentationslinien ergibt die vorgenommene Bewertung, dass keine der bisher vertretenen Argumentationen die Frage abschließend zu beantworten vermag.

Die überzeugenderen Argumente sprechen dafür, dass eine erstmalige Verschriftlichung der 17 Küren und der 24 Landrechte auf Altfriesisch erfolgt ist. Plausibel ist insbesondere die Argumentation Popkemas auf der Grundlage des Textbestands. Für einen lateinischen Urtext spricht nur das Argument des Lateinischen als der dominierenden (Rechts-)Schriftsprache in Westeuropa, welches jedoch durch die friesische Sonderstellung relativiert werden kann. 
Für den Versuch einer Lösung der Frage in der Zukunft sollte beachtet werden, dass die Aussagekraft textinterner Argumente aufgrund der Instabilität der Texte begrenzt ist. Daher ist es zu begrüßen, dass nach der überwiegend am Text ausgetragenen Argumentation Lösungsversuche nunmehr auf der Grundlage anderer Aspekte, z.B. des Textbestandes oder der historischen Frage des Übergangs von Mündlichkeit zu Schriftlichkeit vorgenommen werden.

Zudem lohnt es sich, im Blick zu behalten, dass es sich bei der Frage der Sprache der erstmaligen Verschriftlichung der friesischen Rechtssätze um eine interdisziplinäre Fragestellung handelt. Sofern die Frage der Sprache der allerersten Verschriftlichung überhaupt beantwortet werden kann, liegt der Weg zu ihrer Lösung nach Ansicht der Autorin in einer Kombination verschiedener Ansätze.

\section{LITERATURVERZEICHNIS}

Algra, N. E. 1966. De tekstfiliatie van de 17 Keuren en de 24 Landrechten: een voorbereidend onderzoek (Estrikken 39). Groningen: Frysk Ynst. oan de R.U. to Grins.

Algra, N. E. 1991. Zeventien keuren en Vierentwintig landrechten. Doorn: Graal.

Algra, N. E. 1998. „The Relation between Frisia and the Empire from 8001500 in the Light of the Eighth of the Seventeen Statutes". In: Rolf H. Bremmer Jr et al. (Hg.), Approaches to Old Frisian Philology (Amsterdamer Beiträge zur Älteren Germanistik Band 49, Estrikken 72), Amsterdam: Rodopi, 1-76.

Algra, N. E. 2001. „Grundzüge des friesischen Rechts im Mittelalter“. In: Horst Haider Munske (Hg.), Handbuch des Friesischen/Handbook of Frisian Studies. Tübingen: Niemeyer, 555-570.

Bremmer, Rolf H. Jr. 2004. Hir is eskriven. Lezen en schrijven in de Friese landen rond 1300. Hilversum: Verloren.

Bremmer, Rolf H. Jr. 2009. An Introduction to Old Frisian. History, Grammar, Reader, Glossary. Amsterdam/Philadelphia: Benjamins.

Bremmer, Rolf H. Jr. 2014. „The Orality of Old Frisian Law Texts“. In: Rolf H. Bremmer Jr et al. (Hg.), Directions for Old Frisian Philology (Amsterdamer Beiträge zur Älteren Germanistik Band 73, Estrikken 96), Amsterdam/New York: Rodopi, 1-48. 
Buitenrust Hettema, Foeke. 1892. „Wanneer zijn de oude Friesche wetten opgesteld?" In: Rechtsgeleerd Magazijn. Tijdschrift voor binnen- en buitenlandsche rechtsstudie 11, 341-381.

Heck, Philipp. 1931. Übersetzungsprobleme im frühen Mittelalter. Tübingen: Mohr.

His, Rudolf. 1899. „Die Überlieferung der friesischen Küren und Landrechte“. In: Zeitschrift der Savigny-Stiftung für Rechtsgeschichte. Germanistische Abteilung 20, 39-114.

Hoekstra, J. 1940. Die gemeinfriesischen siebzehn Küren. Friesch-Saksische Bibliotheek 6. Assen: Gorcum.

Hoekstra, J. 1950. De eerste en de tweede Hunsinger Codex. 's-Gravenhage: Nijhoff.

Johnston, Thomas L.B. 1998. „Old Frisian Law and the Frisian Freedom Ideology: Text and Manuscript Composition as a Marketing Device". In: Rolf H. Bremmer Jr et al. (Hg.), Approaches to Old Frisian Philology (Amsterdamer Beiträge zur Älteren Germanistik Band 49, Estrikken 72), Amsterdam: Rodopi, 179-214.

Koegel, Rudolf. 1894. Geschichte der deutschen Litteratur bis zum Ausgange des Mittelalters. Bd. 1, Straßburg: Trübner.

Krogmann, Willy und Wilhelm-Christian Kersting. 1972. „Die friesische Vorstufe des „Vetus lus Frisicum“ (17 Küren, 24 Landrechte, allgemeine Bußtaxen"). In: Zeitschrift der Savigny-Stiftung für Rechtsgeschichte, Germanistische Abteilung 89, 33-77.

Markey, T. L. 1981. Frisian. Trends in Linguistics. State of the Art Reports 13. Den Haag: Mouton.

Popkema, Anne Tjerk. 2005. „In reaksje op Rolf Bremmer syn Hir is eskriven“. In: It Beaken 67, 102-117.

Popkema, Anne Tjerk. 2014. „Old Frisian: a Legal Language in Principle“. In: Rolf H. Bremmer Jr et al. (Hg.), Directions for Old Frisian Philology (Amsterdamer Beiträge zur Älteren Germanistik Band 73, Estrikken 96), Amsterdam/New York: Rodopi, 369-395.

Richthofen, Karl Freiherr von. 1880. Untersuchungen über Friesische Rechtsgeschichte: Erste Abhandlung. Upstalsbom, Freiheit und Grafen in Friesland. Theil 1. Berlin: Hertz.

Roeleveld, Annelies. 1990. „Providentia et Plicht: The Old Frisian Words in the Latin Texts of the Hunsingo manuscripts“. In: Rolf H. Bremmer Jr et al. (Hg.), Aspects of Old Frisian Philology (Amsterdamer Beiträge zur Älteren Germanistik Band 31/32, Estrikken 69), Amsterdam/Atlanta: Rodopi, 371-390. 
Schröder, Richard und Eberhard Frh. v. Künßberg. 1919. Lehrbuch der deutschen Rechtsgeschichte Bd. 1, 6. Aufl. Leipzig: Veit.

Siebs, Theodor. 1902. „Friesische Literatur“. In: Paul, Herrmann, Grundri $\beta$ der germanischen Philologie Bd. 2, 2. Auflage Straßburg: Trübner, 521554.

Sievers, Eduard. 1919. Metrische Studien IV. Die altschwedischen Upplandslagh nebst Proben formverwandter germanischer Sagdichtung Teil 1. Abhandlungen der Philologisch-Historischen Klasse der Sächsischen Akademie der Wissenschaften. Leipzig: Teubner.

Sytsema, Johanneke. 1998. De 17 Keuren en de 24 Landrechten in de Ommelander rechtshandschriften. Amsterdam: VU Uitg. 